TITLE:

\title{
Significance of Ser-188 in human mitochondrial NAD kinase as determined by phosphomimetic and phosphoresistant amino-acid substitutions.
}

\section{AUTHOR(S):}

Kawabata, Yutaka; Murata, Kousaku; Kawai, Shigeyuki

\section{CITATION:}

Kawabata, Yutaka ...[et al]. Significance of Ser-188 in human mitochondrial NAD kinase as determined by phosphomimetic and phosphoresistant amino-acid substitutions. .

Biochemical and biophysical research communications 2015, 468(4): 691-695

ISSUE DATE:

2015-12-25

URL:

http://hdl.handle.net/2433/210210

\section{RIGHT:}

(c) 2015. This manuscript version is made available under the CC-BY-NC-ND 4.0 license

http://creativecommons.org/licenses/by-nc-nd/4.0/; The full-text file will be made open to the public on 25 December 2016 in accordance with publisher's 'Terms and Conditions for Self-Archiving'.; This is not the published version. Please cite only the published version.; この論文は出版社版でありません。引用の際には出版社版をご確認ご利用ください。 
Significance of Ser-188 in human mitochondrial NAD kinase as determined by phosphomimetic and phosphoresistant amino-acid substitutions

Yutaka Kawabata ${ }^{1}$, Kousaku Murata ${ }^{2}$, Shigeyuki Kawai ${ }^{1, *}$

${ }^{1}$ Laboratory of Basic and Applied Molecular Biotechnology, Division of Food Science and Biotechnology, Graduate School of Agriculture, Kyoto University, Uji, Kyoto, Japan

${ }^{2}$ Faculty of Science and Engineering, Department of Life Science, Setsunan University, Neyagawa, Osaka, Japan

* Corresponding author. Laboratory of Basic and Applied Molecular Biotechnology, Division of Food Science and Biotechnology, Graduate School of Agriculture, Kyoto University, Gokasyo, Uji, Kyoto, 611-0011, Japan.

E-mail address: $\quad$ kawais@kais.kyoto-u.ac.jp (S. Kawai)

Abbreviations: NADK, NAD kinase; CD, Circular dichroism. 


\section{ABSTRACT}

Human mitochondrial NAD kinase is a crucial enzyme responsible for the synthesis of mitochondrial $\mathrm{NADP}^{+}$. Despite its significance, little is known about the regulation of this enzyme in the mitochondria. Several putative and known phosphorylation sites within the protein have been found using phosphoproteomics, and here, we examined the effect of phosphomimetic mutations at six of these sites. The enzymatic activity was downregulated by a substitution of an Asp residue at Ser-289 and Ser-376, but not a substitution of Ala, suggesting that the phosphorylation of these residues downregulates the enzyme. Moreover, the activity was completely inhibited by substituting Ser-188 with an Asp, Glu, or in particular Ala, which highlights two possibilities: first, that Ser-188 is critical for catalytic activity, and second, that phosphorylation of Ser-188 inhibits the activity. Ser-188, Ser-289, and Ser-376 were found to be highly conserved in the primary structures of mitochondrial NAD kinase homologs in higher animals. Moreover, Ser-188 has been frequently detected in human and mouse phosphorylation site studies, whereas Ser-289 and Ser-376 have not. Taken together, this indicates that Ser-188 (and perhaps the other residues) is an important phosphorylation site that can downregulate the NAD kinase activity of this critical enzyme.

Key words: NAD kinase, mitochondria, phosphomimetic mutation, phosphorylation, phosphoproteomics. 


\section{Introduction}

NADP $\left(\mathrm{NADP}^{+}\right.$and its reduced form NADPH) is involved in anabolic reactions, as well as in the defense against oxidative stress. It also functions as a substrate in the synthesis of NAADP, a potent intracellular calcium messenger $[1,2]$. NAD kinase (NADK; EC 2.7.1.23), the sole NADP ${ }^{+}$-biosynthetic enzyme known to catalyze the phosphorylation of $\mathrm{NAD}^{+}$into $\mathrm{NADP}^{+}$using $\mathrm{ATP}$, plays a vital role in the cell. There are two types of NADK in human cells: cytosolic NADK (cytNADK) $[3,4,5,6]$ and mitochondrial NADK (mitNADK) $[7,8,9]$, with mitNADK having a much lower $K_{\mathrm{m}}$ (for $\left.\mathrm{NAD}^{+}, 22 \mu \mathrm{M}\right)$ and activity $\left(V_{\max }, 0.091 \mathrm{U} / \mathrm{mg}\right)$ than cytNADK $\left(K_{\mathrm{m}}\right.$ for $\mathrm{NAD}^{+}, 1.07 \mathrm{mM} ; V_{\max }, 18.5 \mathrm{U} / \mathrm{mg}$ ) [7]. A homozygous nonsense mutation in the gene encoding the human mitNADK causes dienoyl-CoA reductase deficiency with hyperlysinemia and a failure to thrive [9], emphasizing the critical role of mitNADK. Despite the importance of both NADKs, little is known about how they are regulated in vivo.

Phosphorylation is a widespread mechanism for controlling the function of enzymes. Recently, Love et al. reported that the N-terminal portion, in particular Ser-64, of human cytNADK is phosphorylated in a $\mathrm{Ca}^{2+} / \mathrm{CaM}$-dependent manner [4]. However, recombinant purified cytNADK mutants with a phosphomimetic (S64D) or a phosphoresistant (S64A) amino-acid substitution at Ser-64 have similar levels of enzymatic activity [4]. Moreover, in vitro phosphorylation of wild-type and S64A cytNADK does not change catalytic activity [4]. Therefore, the physiological role of 
human cytNADK phosphorylation remains to be clarified.

Mitochondria are the major sites of reactive oxygen species (ROS) generation, and mitochondrial ROS levels are tightly regulated, because they have critical roles in the adaptation to hypoxia, autophagy, immunity, differentiation, and probably aging [10]. Because NADPH also participates in the regulation of mitochondrial ROS levels [10], the function of mitNADK is most likely tightly regulated in mitochondria in response to intracellular and extracellular signals. Phosphoproteomics has revealed several phosphorylation sites in mitNADK from humans $[11,12,13]$, mice $[14,15,16]$, and rats $[17,18]$, which are summarized in PhosphoSitePlus (Q4G0N4, http://www.phosphosite.org) (Fig. 1, Table 1).

To elucidate the role of human mitNADK phosphorylation, we introduced several phosphomimetic amino-acid substitutions into human mitNADK and measured the NADK activity of the purified enzymes. We expected that a few of the phosphomimetic mutations would increase activity, because the activity of mitNADK is very low relative to that of cytNADK [7]. However, we found that two phosphomimetic mutations at Ser-289 and Ser-376 downregulated activity and one at Ser-188 abolished its activity altogether, implying that these phosphorylation events actually downregulate this enzyme in mitochondria.

\section{Materials and methods}

\subsection{Plasmid and expression}


Phosphomimetic and phosphoresistant mitNADKs were constructed with PCR using pMK3272 as a template. pMK3272 is the expression plasmid pQE-80L (Qiagen, Venlo, Netherlands) carrying a human mitNADK gene [7]. This gene encodes $\Delta 62$ mitNADK, which lacks the $62 \mathrm{~N}$-terminal amino acids including the mitochondrial targeting sequence [7] (Fig. 1). The expression of wild-type, phosphomimetic (substitution of Ser to Asp or Glu), and phosphoresistant (substitution of Ser to Ala) mitNADK was conducted as described [7]. $\triangle 62$ mitNADK is expressed from pMK3272 as an N-terminal His-Tag $\left({ }^{1}\right.$ MRGSHHHHHHGS $\left.{ }^{12}\right)$-fusion protein. Purification of the expressed protein was performed using a TALON Metal Affinity Resin column (Clontech, Otsu, Japan) [7]. Washing was done with $10 \mathrm{mM}$ Tris-HCl (pH 8.0), 300 $\mathrm{mM} \mathrm{NaCl}$, and $30 \mathrm{mM}$ neutralized imidazole, and elution was done with $10 \mathrm{mM}$ Tris- $\mathrm{HCl}(\mathrm{pH} 8.0), 300 \mathrm{mM} \mathrm{NaCl}$, and $150 \mathrm{mM}$ neutralized imidazole. The fractions containing the purified enzymes were pooled and stored at $4{ }^{\circ} \mathrm{C}$.

\subsection{Assay}

NADK activity was assayed at $37^{\circ} \mathrm{C}$ by the continuous method and the stop method, as described previously [7]. Briefly, NADPH formation was continuously measured at $A_{340}$ in a reaction mixture $(1.0 \mathrm{~mL})$ containing $5.0 \mathrm{mM} \mathrm{NAD}{ }^{+}, 5.0 \mathrm{mM}$ ATP, 5.0 mM glucose 6-phosphate, $0.5 \mathrm{U}$ glucose 6-phosphate dehydrogenase (Sigma, St. Louis, MD), $5 \mathrm{mM} \mathrm{MgCl}$, $100 \mathrm{mM}$ Tris- $\mathrm{HCl}$ (pH 8.0), and an appropriate 
concentration of purified protein. In the stop method, glucose 6-phosphate dehydrogenase was diluted with $50 \%$ glycerol to reduce background. One unit of enzyme activity was defined as $1.0 \mu \mathrm{mol} \mathrm{NADP}{ }^{+}$produced in $1 \mathrm{~min}$ at $37^{\circ} \mathrm{C}$; specific activity was expressed in U/mg protein, unless otherwise stated. The protein concentration of the purified $\triangle 62$ mitNADK and its mutants was determined using $A_{280}$ and a molecular coefficient that was calculated using the ExPASy ProtParam tool [19]. The molecular mass of $\Delta 62$ mitNADK (plus the N-terminal His-Tag) is $44,726.4$, and the molecular coefficient is $50,880 \mathrm{M}^{-1} \mathrm{~cm}^{-1}$ ( $\triangle 62$ mitNADK of $A_{280}=1.138$ corresponds to $1 \mathrm{mg} / \mathrm{ml})$.

\subsection{Circular dichroism (CD)}

The purified mitNADK (wild type), phosphomimetic mitNADK (S188D), and phosphoresistant mitNADK (S188A) were dialyzed against $10 \mathrm{mM}$ Tris- $\mathrm{HCl}(\mathrm{pH} 8.0)$ containing $300 \mathrm{mM} \mathrm{NaCl}$. This dialysis external solution was prepared with ultra-pure water. CD of the purified enzyme was analyzed with a CD spectrometer (J-720 Spectropolarimeter, Jasco, Tokyo, Japan). The control was $10 \mathrm{mM}$ Tris-HCl (pH 8.0) containing $300 \mathrm{mM} \mathrm{NaCl}$. Conditions were as follows: protein concentrations, $0.1-0.2 \mathrm{mg} / \mathrm{ml}$; quartz cell length, $0.01 \mathrm{~cm}$; scan range, 190-260 $\mathrm{nm}$; resolution, $0.1 \mathrm{~nm}$; band length, $1 \mathrm{~nm}$; scan speed, $10 \mathrm{~nm} / \mathrm{min}$; cumulative number, 3 times; and sample volume, $65 \mu \mathrm{L}$. 


\section{Results and discussion}

\subsection{Activity of phosphomimetic mitNADK proteins}

Phosphoproteomics has reveled several phosphorylation sites within the mitNADK of humans [11,12,13], mice $[14,15,16]$, and rats $[17,18]$, which are summarized in PhosphoSitePlus (Q4G0N4, http://www.phosphosite.org) (Fig. 1, Table 1). Among the sites, we focused on Ser and Thr residues, especially on the residues that had been identified as phosphorylation sites in human mitNADK: Thr-183, Ser-188, Ser-345, and Ser-367 [11,12,13]. The detected count for Ser-188 was 207, whereas the counts for Ser-183, Ser-345, and Ser-367 were 6, 1, and 2 in the mass spectrometric analysis conducted by Bian et al. [11], implying that Ser-188 is relatively frequently phosphorylated.

We constructed expression plasmids for the human phosphomimetic mitNADK mutants T813D, S188D, S289D, S294D, S345D, T357D, S363D, S367D, and S376D. These mitNADK mutants were expressed in E. coli and purified (Fig. S1). The phosphomimetic mitNADK S188D, S289D, S345D, S363D, S367D, and S376D were successfully purified. Phosphomimetic mitNADK S183D failed to express, and two other mutants, T357D and S294D, were difficult to express in large quantity and to purify.

We expected that some phosphomimetic mutations would increase activity, because the activity of mitNADK is low relative to that of cytNADK [7]. However, the 
activity of mitNADK S289D and S376D was lower than that of wild type, and the activity of mitNADK S188D was not detected, although mitNADK S363D, S345D, and S367D had the same activity as wild-type mitNADK (Fig. 2A). mitNADK S289E and S188E were also purified (Fig. S1). Again, the activity of mitNADK S289E was lower than that of wild type, and the activity of mitNADK S188E was not detected (Fig. 2A).

To rule out the possibility that Ser-289, Ser-376, and Ser-188 had a significant role in the catalytic activity itself, not in the regulation of the enzyme, we also constructed and purified phosphoresistant mitNADK S188A, S289A, and S376A (Fig. S2). The activity of mitNADK S376A was similar to that of wild type, whereas the activity of mitNADK S289A was 2-fold higher than that of the wild type (Fig. 2B), indicating that the phosphorylation of Ser-376 and Ser-289 reduces activity. The activity of mitNADK S188A was again not detected (Fig. 2B). The purified enzymes (wild-type mitNADK, mitNADK S188D, and mitNADK D188A) had approximately the same CD spectrum (Fig. S3), indicating that the substitutions of Asp or Ala into Ser-188 caused no noticeable structural change. These data highlight two possibilities: first, Ser-188 is critical for catalytic activity, and second, phosphorylation of Ser-188 completely inhibits this activity.

\subsection{Conservation of the investigated Ser residues in the primary structure}

NADKs can be roughly divided into two groups: human mitNADK homologs and human cytNADK homologs. Moreover, mitNADK homologs have sequence 
similarity with plant NADK3 homologs and can be further divided into animal and protist mitNADK homologs [7]. The three key motifs (GGDG, NE/D, and NADK conserved motifs) are conserved in the three groups (mitNADK homologs, plant NADK3 homologs, and cytNADK homologs), and two motifs (motif $1 / 2$ and the C5orf33 additional region) are not found in cytNADK homologs (Fig. 1) [7]. Thr-183 and Ser-188 are found in motif 1/2, and the residues Ser-345, Thr-357, Tyr-360, and Ser-363 are located in the C5orf33 additional region that is specific to mitNADK homologs (Fig. 1).

The conservation of these residues in animal and protist mitNADK homologs and plant NADK3 homologs is listed in Table 2. Multiple alignments of the representative primary structures of mitNADK homologs are shown in Fig. S4. Thr-183, Ser-188, and Ser-289 are highly conserved in animal mitNADK homologs including mouse mitNADK and plant NADK3 homologs (Table 3). Ser-376 is highly conserved $(100 \%)$ in the 62 mitNADK homologs from higher animals (mammals, birds, reptiles, and fishes). Thr-183 and Ser-188 are also found in protist mitNADK homologs from Apicomplexa including the genera Plasmodium, Theileria, and Babesia (Table 3). Earlier, we demonstrated that phosphomimetic mutation of the highly conserved Ser-188 abolished mitNADK activity, and mutation of the conserved Ser-376 and the highly conserved Ser-289 downregulated this activity (Fig. 2).

However, although Ser-183 and Ser-188 were frequently detected as phosphorylation sites, Ser-289 and Ser-376 were not; phosphorylation of Ser-183 and Ser-188 was detected in human mitNADK in two independent experiments $[11,12,13]$. 
Phosphorylation of Ser-188 was detected in mouse mitNADK in three independent experiments $[14,15,16]$, and the phosphorylation of Ser-183 was detected in mouse in one experiment [15]. However, phosphorylation of Ser-289 and Ser-376 was only detected once in mouse and rat mitNADKs, respectively (Table 1) [18]. Taken together, phosphorylation of Ser-188, and perhaps the other sites, could be a significant mechanism for downregulating mitNADK activity.

\section{Acknowledgements}

This work is supported by the Japan Foundation for Applied Enzymology, a research encouragement award, The Sumitomo Foundation, Fundamental Scientific Research Grant-in-Aid Grant Number 140737, and JSPS KAKENHI Grant Number $15 \mathrm{~K} 07387$. 


\section{References}

[1] C.E. Outten, V.C. Culotta, A novel NADH kinase is the mitochondrial source of NADPH in Saccharomyces cerevisiae, EMBO J. 22 (2003) 2015-2024.

[2] R. Aarhus, R.M. Graeff, D.M. Dickey, T.F. Walseth, H.C. Lee, ADP-ribosyl cyclase and CD38 catalyze the synthesis of a calcium-mobilizing metabolite from NADP, J. Biol. Chem. 270 (1995) 30327-30333.

[3] N. Pollak, M. Niere, M. Ziegler, NAD kinase levels control the NADPH concentration in human cells, J. Biol. Chem. 282 (2007) 33562-33571.

[4] N.R. Love, N. Pollak, C. Dolle, M. Niere, Y.Y. Chen, P. Oliveri, E. Amaya, S. Patel, M. Ziegler, NAD kinase controls animal NADP biosynthesis and is modulated via evolutionarily divergent calmodulin-dependent mechanisms, Proc. Natl. Acad. Sci. U. S. A. 112 (2015) 1386-1391.

[5] F. Lerner, M. Niere, A. Ludwig, M. Ziegler, Structural and functional characterization of human NAD kinase, Biochem. Biophys. Res. Commun. 288 (2001) 69-74.

[6] K. Ohashi, S. Kawai, M. Koshimizu, K. Murata, NADPH regulates human NAD kinase, a NADP ${ }^{+}$-biosynthetic enzyme, Mol. Cell. Biochem. 355 (2011) 57-64.

[7] K. Ohashi, S. Kawai, K. Murata, Identification and characterization of a human mitochondrial NAD kinase, Nat. Commun. 3 (2012) 1248 doi: 1210.1038/ncomms2262.

[8] R. Zhang, MNADK, a novel liver-enriched mitochondrion-localized NAD kinase, 
Biol Open 2 (2013) 432-438.

[9] S.M. Houten, S. Denis, H. te Brinke, A. Jongejan, A.H.C. van Kampen, E.J. Bradley, F. Baas, R.C.M. Hennekam, D.S. Millington, S.P. Young, D.M. Frazier, M. Gucsavas-Calikoglu, R.J.A. Wanders, Mitochondrial NADP(H) deficiency due to a mutation in NADK2 causes dienoyl-CoA reductase deficiency with hyperlysinemia, Hum. Mol. Genet. 23 (2014) 5009-5016.

[10] L.A. Sena, N.S. Chandel, Physiological roles of mitochondrial reactive oxygen species, Mol. Cell 48 (2012) 158-167.

[11] Y. Bian, C. Song, K. Cheng, M. Dong, F. Wang, J. Huang, D. Sun, L. Wang, M. Ye, H. Zou, An enzyme assisted RP-RPLC approach for in-depth analysis of human liver phosphoproteome, J. Proteomics 96 (2014) 253-262.

[12] G. Han, M. Ye, H. Liu, C. Song, D. Sun, Y. Wu, X. Jiang, R. Chen, C. Wang, L. Wang, H. Zou, Phosphoproteome analysis of human liver tissue by long-gradient nanoflow LC coupled with multiple stage MS analysis, Electrophoresis 31 (2010) 1080-1089.

[13] K. Sharma, R.C. D'Souza, S. Tyanova, C. Schaab, J.R. Wisniewski, J. Cox, M. Mann, Ultradeep human phosphoproteome reveals a distinct regulatory nature of Tyr and Ser/Thr-based signaling, Cell Rep. 8 (2014) 1583-1594.

[14] J.T. Wilson-Grady, W. Haas, S.P. Gygi, Quantitative comparison of the fasted and re-fed mouse liver phosphoproteomes using lower $\mathrm{pH}$ reductive dimethylation, Methods 61 (2013) 277-286.

[15] P.A. Grimsrud, J.J. Carson, A.S. Hebert, S.L. Hubler, N.M. Niemi, D.J. Bailey, A. 
Jochem, D.S. Stapleton, M.P. Keller, M.S. Westphall, B.S. Yandell, A.D. Attie, J.J. Coon, D.J. Pagliarini, A quantitative map of the liver mitochondrial phosphoproteome reveals posttranslational control of ketogenesis, Cell Metab. $16(2012) 672-683$.

[16] J.C. Trinidad, D.T. Barkan, B.F. Gulledge, A. Thalhammer, A. Sali, R. Schoepfer, A.L. Burlingame, Global Identification and characterization of both o-glcNAcylation and phosphorylation at the murine synapse, Mol. Cell. Proteomics 11 (2012) 215-229.

[17] A. Lundby, A. Secher, K. Lage, N.B. Nordsborg, A. Dmytriyev, C. Lundby, J.V. Olsen, Quantitative maps of protein phosphorylation sites across 14 different rat organs and tissues, Nat. Commun. 3 (2012).

[18] J.D. Hoffert, T. Pisitkun, G.H. Wang, R.F. Shen, M.A. Knepper, Quantitative phosphoproteomics of vasopressin-sensitive renal cells: Regulation of aquaporin-2 phosphorylation at two sites, Proc. Natl. Acad. Sci. U. S. A. 103 (2006) 7159-7164.

[19] M.R. Wilkins, E. Gasteiger, A. Bairoch, J.C. Sanchez, K.L. Williams, R.D. Appel, D.F. Hochstrasser, Protein identification and analysis tools in the ExPASy server, Methods Mol. Biol. 112 (1999) 531-552.

[20] S.F. Altschul, W. Gish, W. Miller, E.W. Myers, D.J. Lipman, Basic local alignment search tool, J. Mol. Biol. 215 (1990) 403-410.

[21] J.D. Thompson, D.G. Higgins, T.J. Gibson, CLUSTAL W: improving the sensitivity of progressive multiple sequence alignment through sequence 
weighting, position-specific gap penalties and weight matrix choice, Nucleic Acids Res. 22 (1994) 4673-4680. 


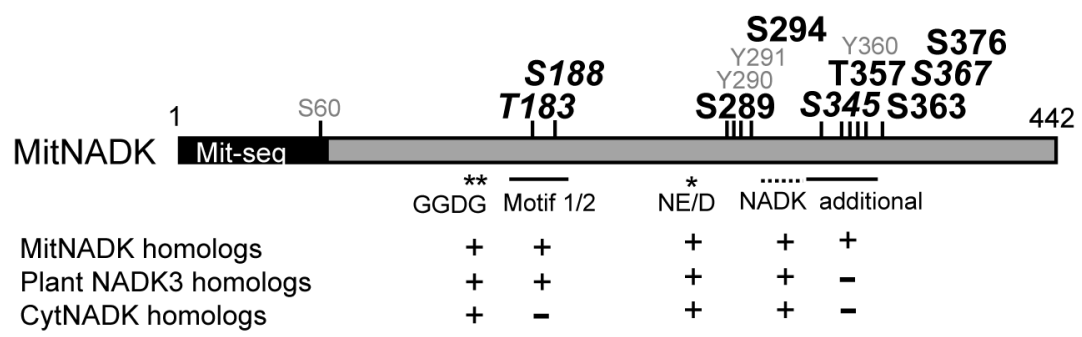

Fig. 1. Putative and detected phosphorylation sites in human mitNADK $[11,12,13,14,15,16,17,18]$, and the structures of NADK homologs. The residues studied here are in bold, and the residues that have been detected in human mitNADK with phosphoproteomics $[11,12,13]$ are in italics (Thr-183, Ser-188, Ser-345, and Ser-367). Recombinant mitNADK ( $\triangle 62$ mitNADK) lacks the mitochondrial targeting sequence (Mit-seq, 62 residues) [7]. Primary structures of mitNADK homologs possess the GGDG motif, motif $1 / 2$, the NE/D short motif, the NADK conserved motif (NADK), and the C5orf3 additional region (additional); in plant NADK3 homologs, only the additional region is not present (-), whereas in cytNADK homologs, motif $1 / 2$ and the additional region are not present [7]. Note that plant NADK3 homologs have inserted sequence (NADK3 additional region consisting of 15 amino acids) between motif 1 and motif $2[7]$. 

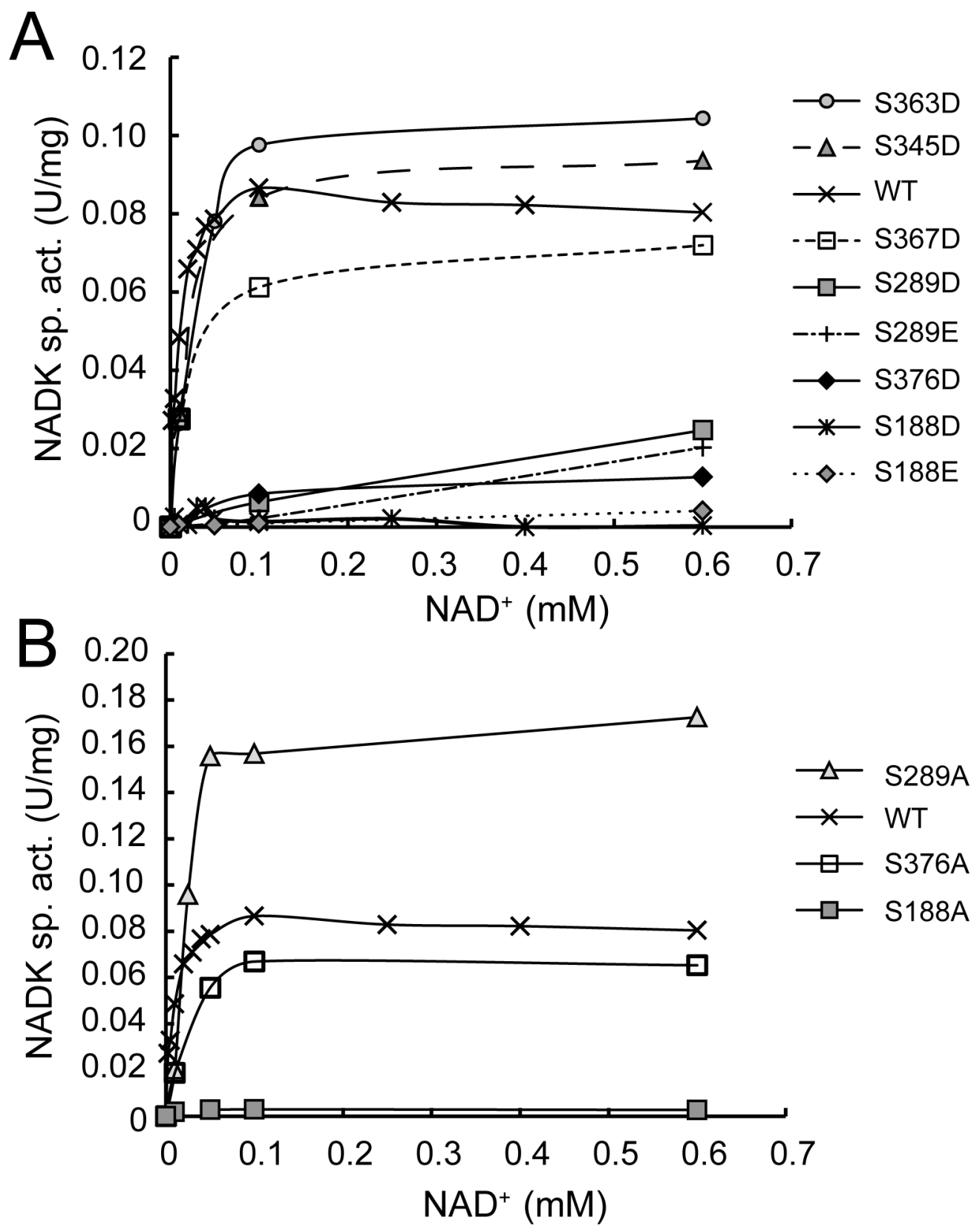

Fig. 2. NADK activity of each (A) phosphomimetic or (B) phosphoresistant mitNADK. 
Table 1. The phosphorylation sites of mitNADK in human, mouse, and rat ${ }^{\mathrm{a}}$

\begin{tabular}{|c|c|c|c|c|}
\hline mitNADK site & $\begin{array}{l}\text { Corresponding } \\
\text { human site }\end{array}$ & Ref & $\begin{array}{l}\text { Disease } \\
\text { tissue studied }\end{array}$ & $\begin{array}{l}\text { Relevant cell } \\
\text { line / cell } \\
\text { type / tissue }\end{array}$ \\
\hline \multicolumn{5}{|l|}{ Human } \\
\hline Thr-183 & Thr-183 & [11] & & liver \\
\hline Ser- $188^{b}$ & Ser- $188^{b}$ & & & \\
\hline Ser-345 & Ser-345 & & & \\
\hline Ser-367 & Ser-367 & & & \\
\hline Thr-183 & Thr-183 & [12] & hepatocellular & hepatocyte- \\
\hline Ser-188 & Ser-188 b & & $\begin{array}{l}\text { carcinoma, } \\
\text { surrounding } \\
\text { tissue }\end{array}$ & liver \\
\hline Ser-367 & Ser-367 & [13] & & $\begin{array}{l}\text { HeLa } \\
\text { (cervical) }\end{array}$ \\
\hline Mouse & & & & liver \\
\hline Ser-48 & Ser-60 & [14] & & \\
\hline Ser-176 & Ser-188 & & & \\
\hline Ser-48 & Ser-60 & [15] & & liver \\
\hline Thr-171 & Thr-183 & & & \\
\hline Ser-176 & Ser- $188^{b}$ & & & \\
\hline Ser-377 & Ser-367 & & & \\
\hline Ser-176 & Ser- $188^{b}$ & [16] & & brain \\
\hline
\end{tabular}




\begin{tabular}{|c|c|c|c|}
\hline Ser-299 & Ser- $289^{b}$ & (2007) & \\
\hline Tyr-300 & Tyr-290 & CST & \\
\hline Tyr-301 & Tyr-291 & Curation & \\
\hline Ser-304 & Ser-294 & Set: 3621 & \\
\hline \multicolumn{4}{|l|}{ Rat } \\
\hline Ser-43 & Ser-60 & {$[17]$} & liver \\
\hline Thr-340 & Thr-357 & {$[18]$} & kidney, inner \\
\hline Tyr-343 & Tyr-360 & & medulla \\
\hline Ser-346 & Ser-363 & & \\
\hline Ser-359 & Ser- $376^{b}$ & & \\
\hline
\end{tabular}


Table 2. Number of homologs in which the phosphorylation site in human mitNADK is conserved. ${ }^{\mathrm{a}, \mathrm{b}}$

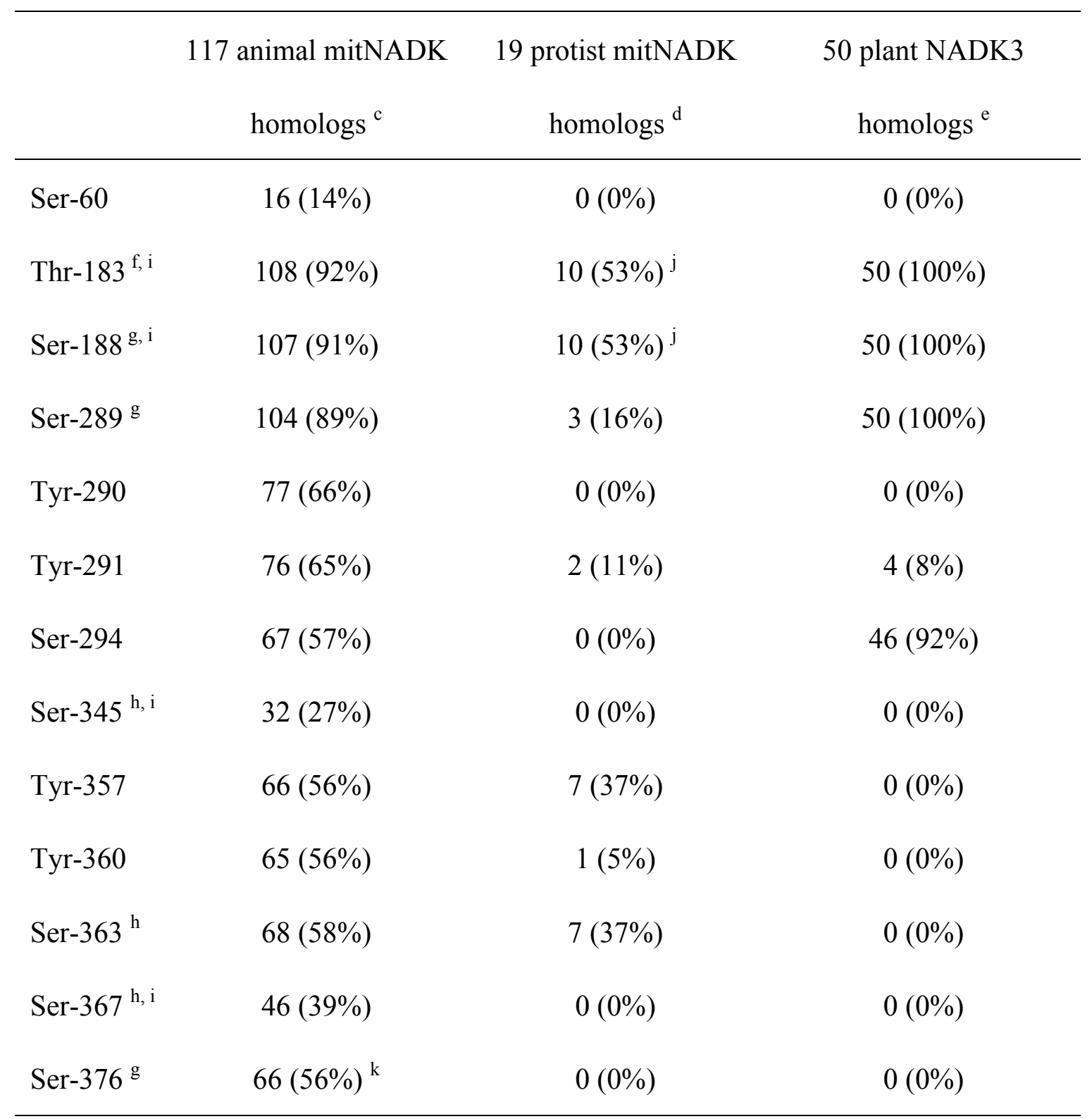

${ }^{\mathrm{a}}$ The sites shown in Fig. 1.

${ }^{\mathrm{b}}$ Blast analysis [20] was conducted using the primary structure of human mitNADK as a query, and the obtained data was aligned using ClustalW [21].

${ }^{\mathrm{c}, \mathrm{d}, \mathrm{e}}$ The homologs with e value $<3 \mathrm{e}-16^{\mathrm{c}},<6 \mathrm{e}-05^{\mathrm{d}}$, and $<0.007^{\mathrm{e}}$. 
${ }^{\mathrm{f}}$ For example, Thr-183 of human mitNADK is conserved as a Ser or Thr residue in 108 animal mitNADK homologs.

g, h Phosphomimetic substitutions of these residues into Asp or Glu downregulated the activity $^{\mathrm{g}}$ or had no effect on activity ${ }^{\mathrm{h}}$.

${ }^{\mathrm{i}}$ The residues had been identified as phosphorylation sites in human mitNADK with phosphoproteomics $[11,12,13]$.

${ }^{\mathrm{j}}$ All 10 homologs belong to protist mitNADK homologs from Apicomplexa including the genera Plasmodium, Theileria, and Babesia.

${ }^{k}$ Ser-376 is highly $(100 \%)$ conserved in the 62 mitNADK homologs from higher animals (mammals, birds, reptiles, and fishes). 


\section{Supporting information for}

Significance of Ser-188 in human mitochondrial NAD kinase as determined by phosphomimetic and phosphoresistant amino-acid substitutions

Yutaka Kawabata, Kousaku Murata, Shigeyuki Kawai

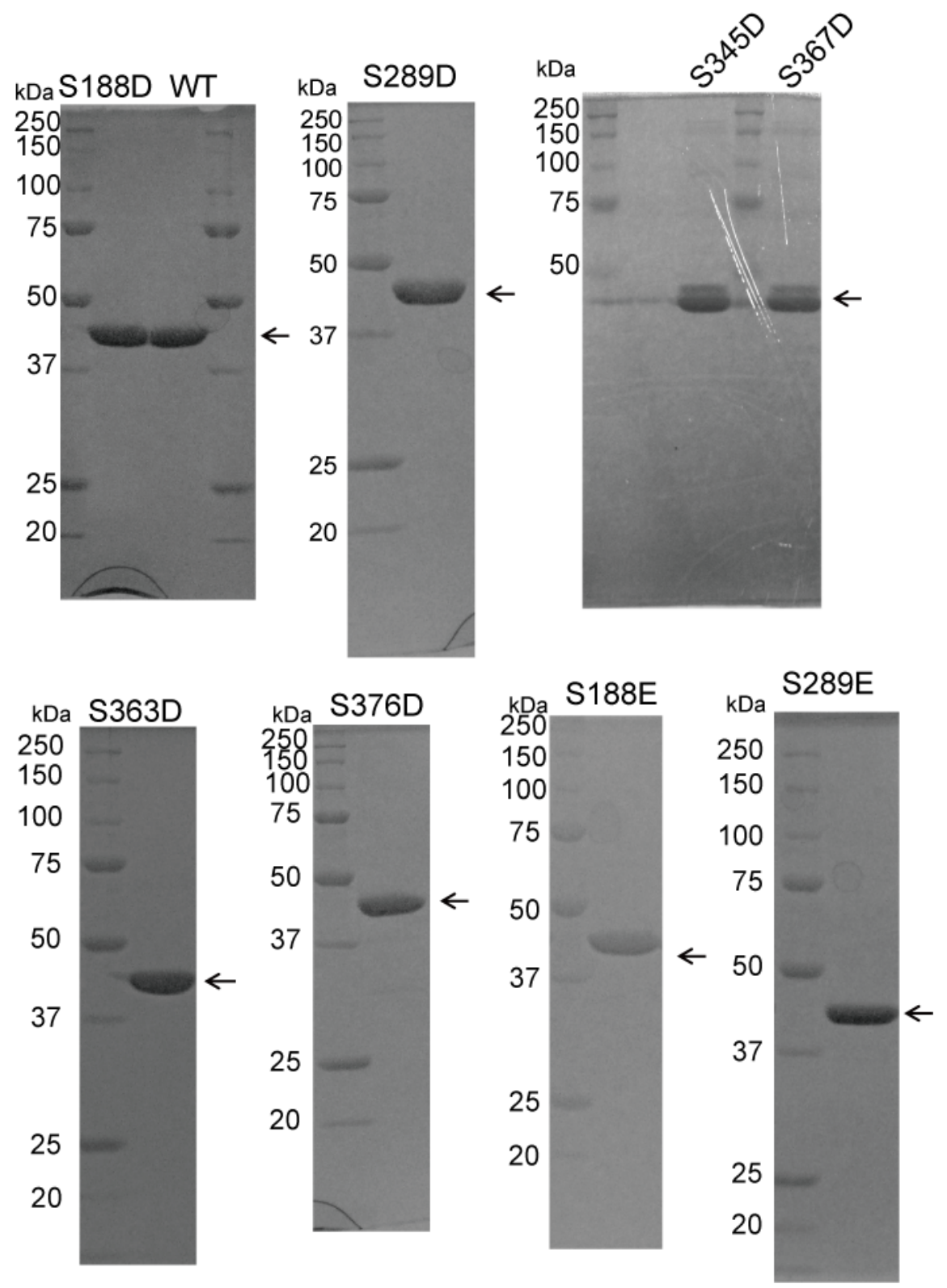

Fig. S1. SDS-PAGE of the purified wild-type (WT) and phosphomimetic mitNADK $(3 \mu \mathrm{g})$. 


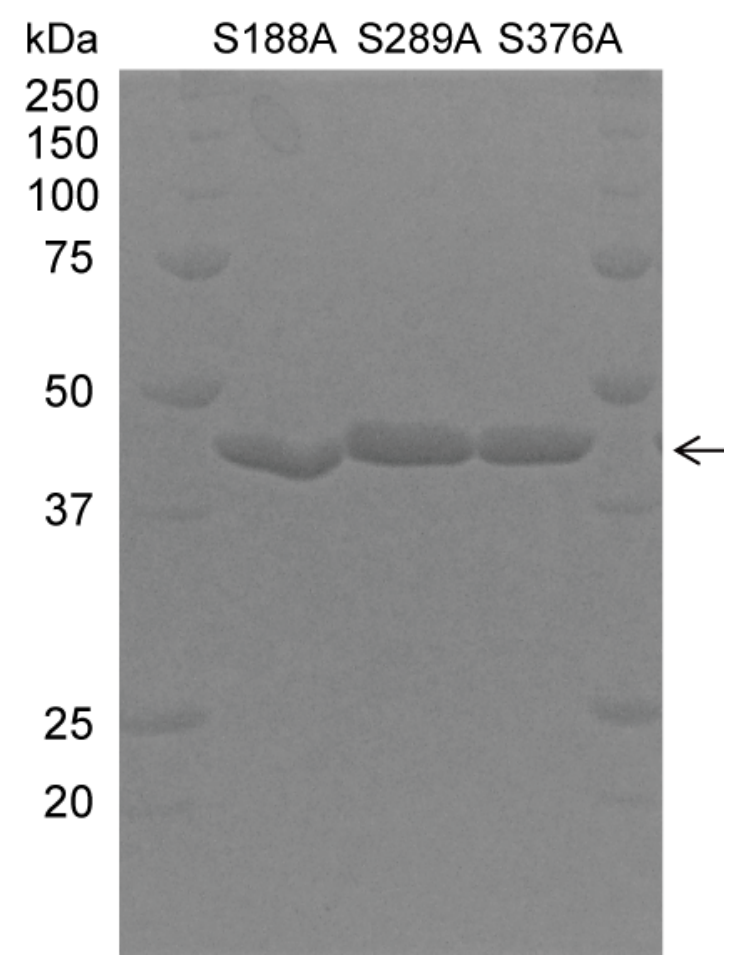

Fig. S2. SDS-PAGE of the purified phosphoresistant mitNADK (3 $\mu \mathrm{g})$. 


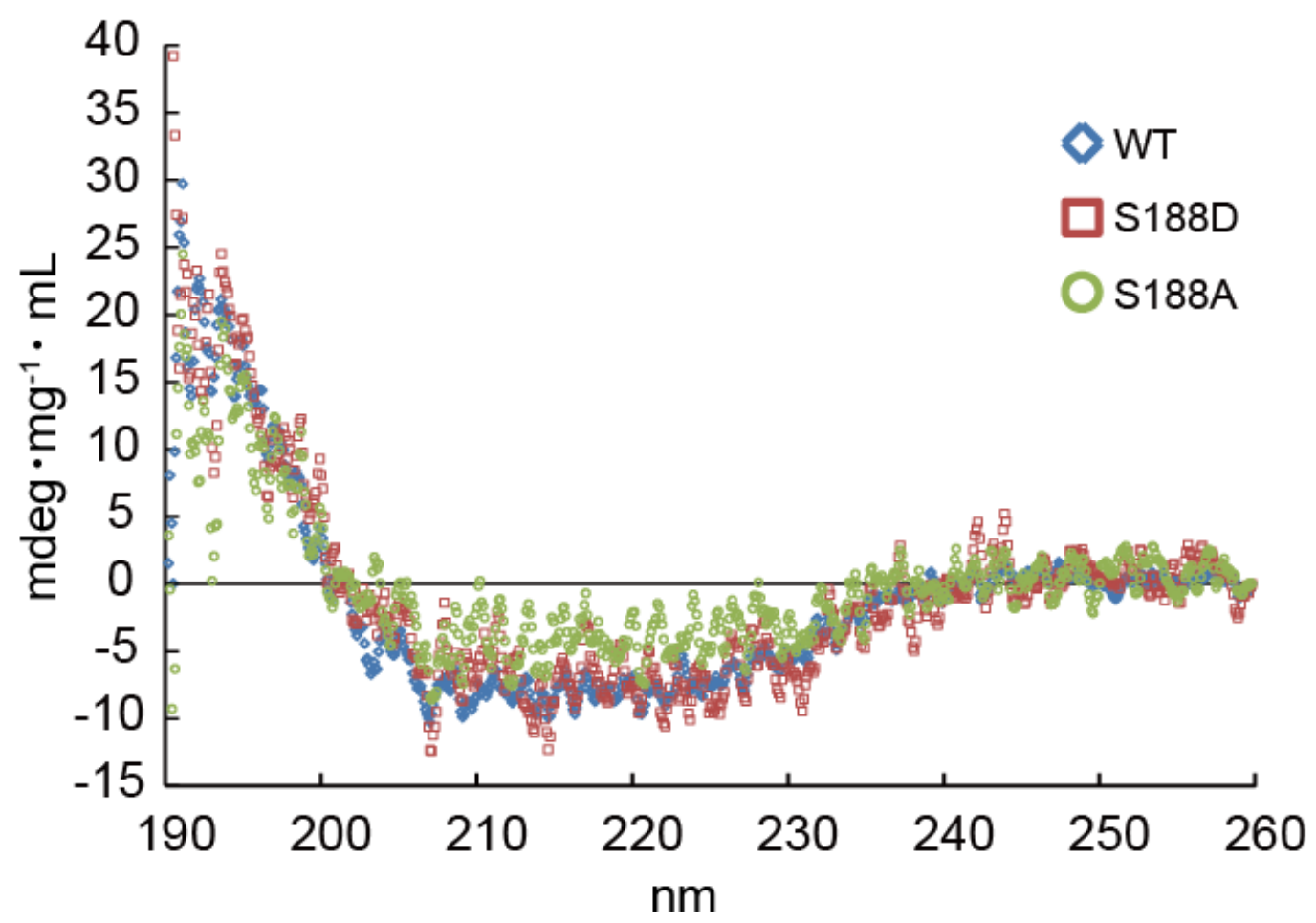

Fig. S3. CD spectrum of the purified enzymes (wild-type [WT] mitNADK, mitNADK S188D, and mitNADK S188A). 
Human mi tNADK Sus scrofa
Bos taurus Rattus norvegicus Ailuropoda melanoleuca Monodelphis domestic
Anolis carolinensis ornithorhynchus anatinus Gallus gallus Xenopus tropicalis Taeniopygia guttata Strongylocentrotus purpuratus Caenorhabditis elegans Branchiostoma floridae Pediculus humanus corporis Caenorhabditis elegans Phytophthora infestans

C5orf33.
Macaca mulatta
Sus scrofa
Bos taurus
Rattus norvegicus
Ailuropoda melanoleuca
Monodelphis domestica
Anolis carolinensis
Ornithorhynchus anatinus
Gallus gallus
Xenopus tropicalis
Taeniopygia guttata
Strongylocentrotus purpuratus
Caenorhabditis elegans
Caenorhabditis briggsae
Branchiostoma floridae
Pediculus humanus corporis
Caenorhabditis elegans
Caenorhabditis briggsae
Phytophthora infestans

\section{GGDG motif Motif 1 Motif 2}

136: VRLVKRREYDEE TVRWADAVIAAGGDGTMLLAASKVLDRLKYVIGVVTDPER 162 GEGHLCLPVRYTHS--FPEALQKFYRGE FRWLWRQRIRLYLEGTGINP :233 36: VRLVKRREYDEE TVRWADAVIAAGGD GTMLLAASKVLDRLKPVIGVN

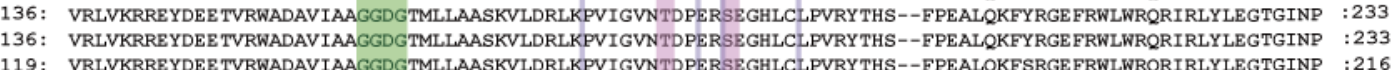
119: VRLVKRREYDEETVRNADAVIAAGGDGTMLLAASKVLDRLKPVIGVNTDPER SEGHLCLPVRYTHS--FPEALQKFSRGEFRWLWRQRIRLYLEGTGINP : 216

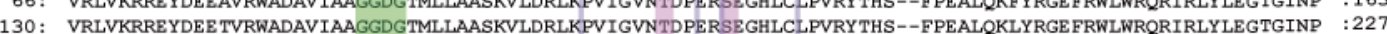

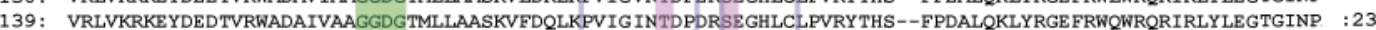
36: VRLVKRREYDEE TVRWADAVIAAGGDGTMLLAASKVLDRLKPVIGVNTDPERSEGHLCLPVRYTHS--FPEALKRLTHGEFRWLWRQRIRLYLEGTGINT : 13 49: VRLVKRRDYNEETVRWADAVISAGGDGTMLLAASKVFDKFKPVIGVNTDPERSEGHLCLPVRYTHS--FPEALQKLYRGE FRWQWRQRIRLYLEGTGINP : 14 106: VRLVKRRDYDEE TVRWADAI ISAGGDGTMLLAASKVQDRFKPVIGVNTDPERSEGHLCLPVRYTWS--FPEALQKLYRGEFRWQWRQRIRLYLEGTGINL : 203 187: VRLVKRREYNEE TVRWADAVISAGGD GTMLLAASKVFDKFKPVIG INTDPERSEGHLCLPVRYTHS--FPEALQKLYRGEFRWQWRQRIRLYLEGTGINT : 28 60: SRLVRREGYTOEAVDWADAVFSAGGDGTFLMASSRVRTKHKPVIG INTDPQGSEGYMCLMRKLPEEN-LAGALKKLFSGNFEWLNRQRIRITVTGDDGIS :250 54: SRLVRRFGYTOEAVDWADAVFSAGGDGTFLMASSKVRTKHKPVIG INTDPQGSEGYMCLMRKLPEEN-LSGALKKLFSGNFEWLYRQRIRI TVTGDDGIS : 252 58: VKVIQKHEYTPEKVNWADVIMSAGGDGTFLMAASHILTRKKPLIGVNTDPSR SEGYLCLPKEYSGK--FSRALDRLLAGKFRWRWRNRIRITLEGYQVSH : 155 130: IRNYNRFNCTHDDIEWCTSVFPIGGDGTFLYAARQISNTDKPVIGFNSDPSR SEGYLCLPKKYSNN--ILDALKKLINGDFRWMFRTRIRVTLNEQYVSC : 227 99: TKVVTR-EQLAQYLPEADLVISAGGDGTFLAAASVUNDNT-PIIG INTDPIGSEGHLCVGGKNPPR----DLIERLVSGNLKWVQRTRIRVTVKESRNSI :192 (504

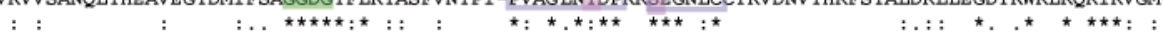

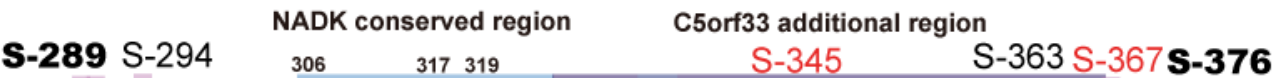

$306 \quad 317319$ 289: SYYYISVDGG-PWEKQKSSGLNLCTGTGSKANSFNINR YATQAVE DVLNIAKRQGNLS---LPLNRELVEKVTNEYNESLLYSPEEPKILFSIRE PIANRT : 384 289: SYYE ISVDDG-PWEKQKSSGLNLCTGTGSKAWSFNINR YATQAVE DVLNIAIRQGNLS---LPLNRELVEKVTNEYNESLLYS SPEEPKILFSIRE PIANRT : 384 272: SYYE ISVDDG-PWEKQKS SGLNLCTGTGSKANSFNINR YAPQAVE DVLNIANRQGNLS---LPLNRELVEKVTNEYNESLLYSPEEPKILFSIRE PIANR: : 384 219: SYYT SVDDG-PWEKOKSSGLNICTGTGSKANSFNINR YATQAVE PVLNIAKRQGNLS---LPLNRELVEKVTNEYNESLLYYSPEEPKILFSIRE PIANRT : 367 283: SYYEISIDDG-PNEKOKSSGLNLCTGTGSKAWSYNINR VATOAVE DVLKIAKROANLD---LPLKKELVEKVTNEYNESLLYSPEE PKMLFSIRE PITNR: : 378 292: SYYEISVDDG-PWEKQKSSGLNICTGTGSKAWSFNINK YANQAVE EILKIAKGYDSLN---LPLSKELIQKVTDGYNESLLYSPEE PKMLFSVRE PISNRT : 387 189: SYYE ISIDDG-PWEKRKS SGLNLCTGTGSKANSYNINR YATQAVE DVLTIAQRQGNLN---IPLSRELIEKVTNEYNDSLLYSPEEPKMLFSIRE PIANRT : 284 274: SYYE ISVDDG-PWEKRKSSGLNVCTGTGSKANSYNINK YANQAVE EILNIAKKHGSLN---MPLNTELVRKVTNAYNESLLYSPEE PKMFFSIRE PIVNRT : 297 340: SYYE I 210: SYYEMSVDDG-PMEKOKSSGVTVSTGTGSSSWSFNINKLSCLSVKDILKISEYSNEE TGSNLATEDCTVERIADRFNSSLLEDGADPRMAYTVRD PVTNRT : 308 338: SYYEIGINDA-QMLKQKS SGITICTGTGSTSWNFNINKLTEQCVQ DLMKIVAEHCNLP-QI PHGDKNAVSE ICTKFNQQLI IDPDRKQLAFSVRD PIFNA: : 435 336: SYYE IGLNDE-QMLKRKS SGI TICTGTGSTSWHFNINKLTEQCVQDLMKIVAQHCNLP-QI PHENKNAVSE ICTKFNQQLIIPPDRRQLAFSVRD PIFNA: : 433 224: SYYELSVDGR-RWEKQKSSGLTVLMRR-----1---10

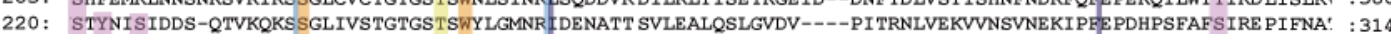
224: STYNISIDDS-OSVKOKSGGLIVSTGTGSTSWYLGMNR FDENATT SVLEALQSLGINV-----PT TRNLVEKVVTTVNEKIPEE PDHPSFAFSIRE PIFNA: : $: 314$ 526: SHYNIGIDQH-QRESHRSSGILMCTGTGSSAWYSSACQ YYREQVA TVLSAMDHTHTNET---------VTELTESINKQNVEPEDSRDMGYVVRE PI INA: : : 615 *:: :: .:***: :

Fig. S4. Multiple alignments of the representative primary structures of mitNADK homologs. Ser-188, Ser-289, and Ser-376, which downregulated NADK activity when they were substituted with phosphomimetic Asp, are in bold. Residues that were detected in human mitNADK are in red. 